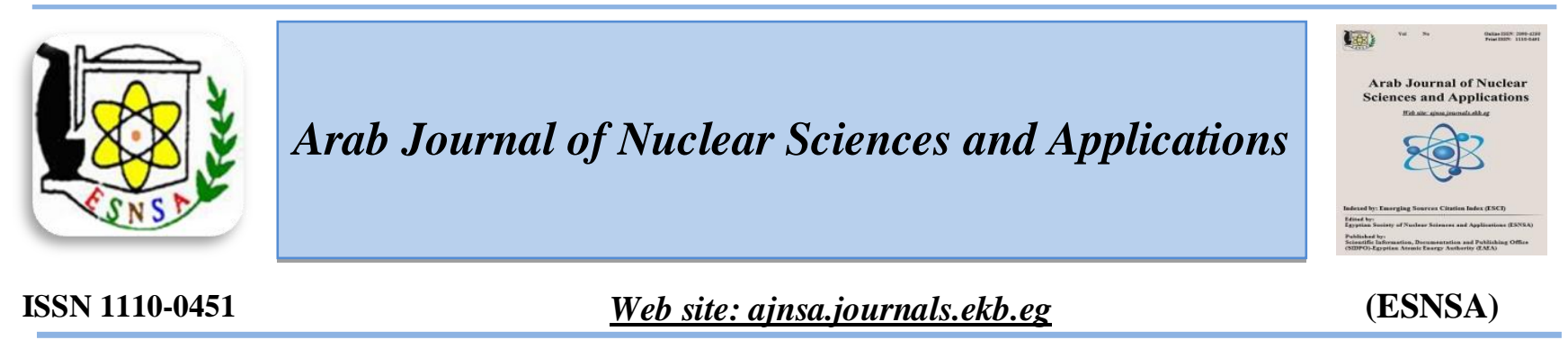

\title{
Prediction of Potential Dose Metrics for the Estimation of Health Effects due to Occupational Exposure in Samalut City, Egypt
}

\author{
M. Moustafa ${ }^{1}$ and I. Balásházy ${ }^{2}$ \\ ${ }^{1}$ Physics Department, Faculty of Sciences, Minia University, El-Minia, Egypt \\ ${ }^{2}$ Centre for Energy Research, Hungarian Academy of Sciences, H-1525 Budapest, Hungary
}

\begin{abstract}
Received $23^{\text {rd }}$ Jan 2019 Potential dose metrics for the estimation of health effects were computed considering a stochastic lung Accepted $3^{\text {rd }}$ Mar 2019 model based on the measured size distribution of elemental aerosols in the industrial area of Samalut city (El-Minia, Egypt). Mass deposition fractions, mass deposition rates and mass deposition density rates were calculated for different physical activity levels: sitting, light and heavy exercise breathing. The highest mass deposition fractions were observed in the extrathoracic region, while deposition in bronchial and acinar regions was consistently smaller irrespective of the chemical species. This difference between extrathoracic and lung regions was further exacerbated in the case of mass deposition density rates due to significant differences in related surface areas. Mass deposition fractions and mass deposition rates increased with airway generation number, reaching a maximum in the acinar region, while mass deposition density rates dropped significantly from the maximum in the large bronchial to the distal acinar airways. Mass deposition rates and deposition density rates increase with physical activity level and thus are highest for heavy exercise breathing conditions. Mass deposition density rates suggest that the bronchial region is the primary target for health effects of inhaled particles.
\end{abstract}

Keywords: Human respiratory tract / Elemental aerosols / Industrial area / Stochastic lung deposition model.

\section{Introduction}

Inhalation is the most common route of entry for industrial aerosols. The assessment of the risk due to inhaled airborne pollutants for different exposed population groups represents one of the main tasks in the field of industrial hygiene and health physics. Many occupational diseases are associated with the deposition of particles within a certain region of the respiratory tract. In general, there is a direct relation between the amount of a toxic dust in the air breathed by a person and the likelihood that the person suffer from pulmonary diseases [1] Particles initially deposited in the lung may then be cleared to the gastro-intestinal tract by mucociliary action or to the bloodstream by diffusion through the alveolar membrane [2], where they are subsequently transported to all organs and tissues of the human body. As a consequence, all human organs may exhibit a toxicological response following inhalation of particulate matter. Health effects of inhaled particulate matter are determined by a complex set of physiological and/or physical, chemical and biological properties of both the respiratory system and the aerosol [2].

Metals are commonly found in almost all sizes of atmospheric particulate matter [3], and when associated with respirable particles lead to increased risks to contract numerous diseases [4,5]. Indeed, there is mounting evidence of detrimental effects caused by air pollutants including trace 
metals [6,7]. Adverse health effects of inhaled particles depend strongly on their size, specific surface area, number and chemical composition that regulate the toxicity of any specific element $[8,9]$.

Iron may cause conjunctivitis, choroiditis, and retinitis if it contacts and remains in the tissues. Chronic inhalation of excessive concentrations of iron oxide fumes or dusts may result in the development of a benign pneumoconiosis, called siderosis. Inhalation of excessive concentrations of iron oxide may enhance the risk of lung cancer development for workers exposed to pulmonary carcinogens $[7,8]$.

Potassium plays an important role in the physical fluid system of humans and it assists nerve functions. An accumulation of potassium in the kidneys may cause kidney malfunctioning, which can disturb the heartbeats. Inhalation of dust containing potassium can irritate the eyes, nose and throat, lungs with sneezing, coughing and sore throat. Higher exposures may cause a buildup of fluid in the lungs, which can lead to death. Skin and eye contact can cause severe burns leading to permanent damage

Although copper is essential for preserving a good health, exposure to higher doses can be harmful. Long-term exposure to copper dust can irritate the nose, mouth, and eyes, and it causes headaches, dizziness, nausea, and diarrhea. Intentional high intakes of copper can cause liver and kidney damage and even death $[7,8]$.

The exposure of workers to high levels of manganese also affects the nervous system. The health effects in this case are represented by behavioral changes and other nervous system's effects, which include movements that may become slow and clumsy. This combination of symptoms, when sufficiently severe, is referred to as "manganism". Other less severe nervous system's effects, such as slower hand movements, have been observed in some workers exposed to lower concentrations at the workplace. The inhalation of a large quantity of dust or fumes containing manganese may cause irritation of the lungs, which could lead to pneumonia [7].

The main target for lead toxicity is the nervous system, both in adults and children. Long-term exposure of adults to lead at work has resulted in decreased performance in some tests that measure functions of the nervous system. Lead exposure may cause weakness in fingers, wrists, or ankles, small increases in blood pressure and may cause anemia. At high levels of exposure, lead can severely damage the brain and kidneys in adults or children, and ultimately it causes death. In the case of pregnant women, high levels of exposure to lead may cause miscarriage.

High calcium intake can cause constipation. It might also interfere with the absorption of Iron and Zinc, though this effect is not well established. High intake of Calcium from supplements, but not foods, has been associated with increased risk of kidney stones occurrence.

The US Environmental Protection Agency (EPA) has concluded that barium is not likely to be carcinogenic to humans following ingestion and that there is insufficient information to determine whether it will be carcinogenic to humans following inhalation exposure. The Department of Health and Human Services (DHHS) and the International Agency for Research on Cancer (IARC) have not classified barium as a carcinogen. Effects of inhaled aerosols depend on the specific chemical species, the concentration, the duration of exposure, and the site of deposition within the lung. Particle size is the most important factor that determines its deposition properties [10]. Nanosized particles are efficiently deposited by diffusional mechanisms in all regions of the lung. Alternatively, the small size facilitates transcytosis across epithelial and endothelial cells into the blood and lymph circulation to reach potentially sensitive target sites such as bone marrow, lymph nodes, spleen, and heart [11]. Epidemiological studies, as well as animal inhalation experiments, support the hypothesis that physical (e.g. particle size, shape, and electric charge) and chemical properties (e.g. solubility or transportability) of single particles are also involved in their potentially toxic, genotoxic, and carcinogenic health effects. For these reasons, more information on both the human exposure to aerosol particles, (including their deposition and clearance) and on the toxicity hazard of the deposited particles, is required [12].

The assessment of health risks resulting from the inhalation of particulate matter in general and of the elements considered in this study in particular requires the calculation of particle deposition in the human lung. Potential dose metrics for the estimation of health effects are (i) mass deposition fractions, i.e. the fraction of the mass of inhaled particles deposited in a given region (or airway 
generation) of the lung during a single breath, (ii) mass deposition rates, i.e. mass deposited in a given region (or airway generation) of the lung per time unit, and (iii) mass deposition density rates, i.e. mass deposition rates per unit surface area, which is equivalent to a "particle dose rate". Therefore, the present work includes the study of particulate matter (PM) because most of the aerosol particles consist of mixed minerals, $\mathrm{Fe}$ is selected from the measured elements due to its high concentration with respect to the other elements. In addition, Fe has a unity enhancement factor which induces a strong effect of a natural component. On the other hand, $\mathrm{Pb}$ is selected because it showed an enhancement factor higher than 10 suggesting that $\mathrm{Pb}$ is emitted mainly from anthropogenic sources [13].

Based on the measured concentrations and size distributions of elemental aerosols and particulate matter (PM) in the industrial area of Samalut city (El-Minia, Egypt) [13], the present study aims to calculate (i) mass deposition fractions of PM, choosing $\mathrm{Pb}$ as an example of anthropogenic element and $\mathrm{Fe}$ as an example of soil- derived element, (ii) corresponding mass deposition rates in the human lung, and (iii) related mass deposition density rates. Deposition calculations were performed for adult males considered under different physical activities, i.e. sitting, light and heavy exercise breathing conditions.

\section{Materials and Methods}

Measurements of elemental aerosol size distributions

Mass size distributions of the elemental aerosols in an industrial area of Samalut city (El-Minia, Egypt) were studied by Moustafa et al. [13], these measurements are briefly summarized below.

To collect the aerosol particles a Berner cascade impactor was used. The impactor operates at a flow rate of $1.7 \mathrm{~m}^{3} \mathrm{~h}^{-1}$. It contains eight size fractionating stages with the cut-off diameters of $82,157,270,650,1110,2350,4250$ and $5960 \mathrm{~nm}$, respectively. Cut-off diameter is defined as the particle size that gives $50 \%$ of the collection efficiency. Samples were collected at an iron and steel quarry close to a cement factory in Samalut city (latitude: $\mathrm{N} 28^{\circ} 18^{\prime} 30^{\prime \prime}$, longitude E $30^{\circ} 42$ $28 ")$.

The samples collected by the low-pressure Berner cascade impactor were analyzed by atomic absorption at the National Institute for Standards (NIS), Egypt. Knowing the mass of the collected particles, the flow rate of the impactor and the sampling time, the specific mass concentrations of the aerosol particles could be determined [13].

Measured particle mass size distributions of the considered aerosols for the study ( $\mathrm{PM}, \mathrm{Pb}$ and $\mathrm{Fe}$ ) are presented in Figure (1).

Modeling the deposition of aerosol particles in the human respiratory tract

An advanced version of stochastic lung deposition model IDEAL [14], originally developed by Koblinger \& Hofmann [15] and Hofmann \& Koblinger [16], was used for calculating the deposition of inhaled particles in the human respiratory system. For modeling purposes, the respiratory system was divided into three regions, namely: extrathoracic (ET), bronchial $(\mathrm{Br})$ and acinar (Ac) region, respectively. The bronchial tree and the acinar region are mode led by a sequence of cylindrical tubes asymmetrically branching at an airway bifurcation into two daughter airways. In the acinar region, more and more hemispherical alveoli cover the walls of the cylindrical airways, until the lung is finally terminated by alveolar sacs, which are completely covered by alveoli Figure (2). On average, airway generations from 1 through 15 typically represent the bronchial region of an adult human, while the acinar region commonly consists of airway generations from 16 through 25 [2]. However, as a result of the asymmetric branching at airway bifurcations, airway generations from 12 to 20 contain both $\mathrm{Br}$ and Ac airways.

In the stochastic lung deposition model, the path of an inhaled particle through the branching airway system is randomly selected by Monte Carlo methods, following the same path during expiration. As a consequence of the variability of the selected sequence of airways, all paths of inspired particles are different from each other, and so are the deposition fractions in the individual airways. Upon expiration, particles follow the same path as during inspiration. By simulating the random paths of many particles, typically of the order of tens or hundreds of thousands, statistical means can be calculated for total, regional, and generational deposition, providing also information on the underlying statistical distributions. 


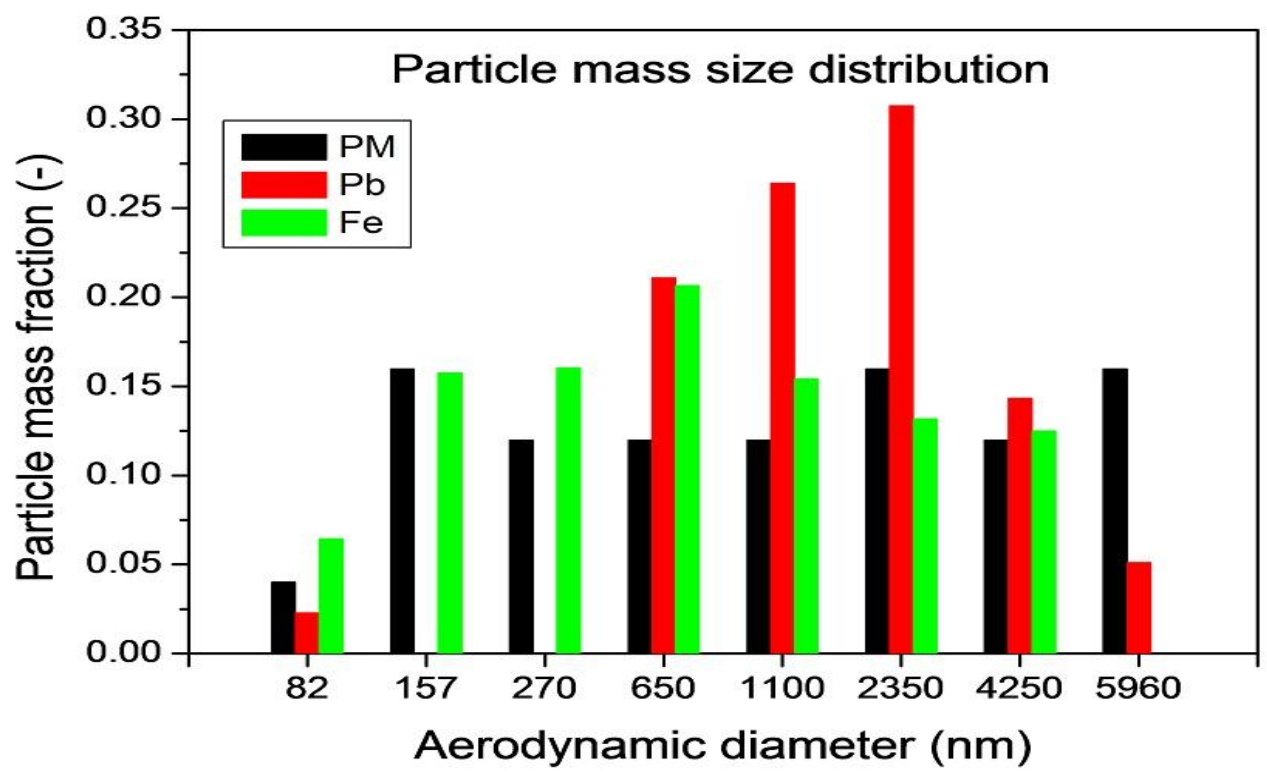

Figure (1): Mass size distributions of PM, Fe and $\mathrm{Pb}$ in the air of the industrial area of Samalut city (El-Minia, Egypt)

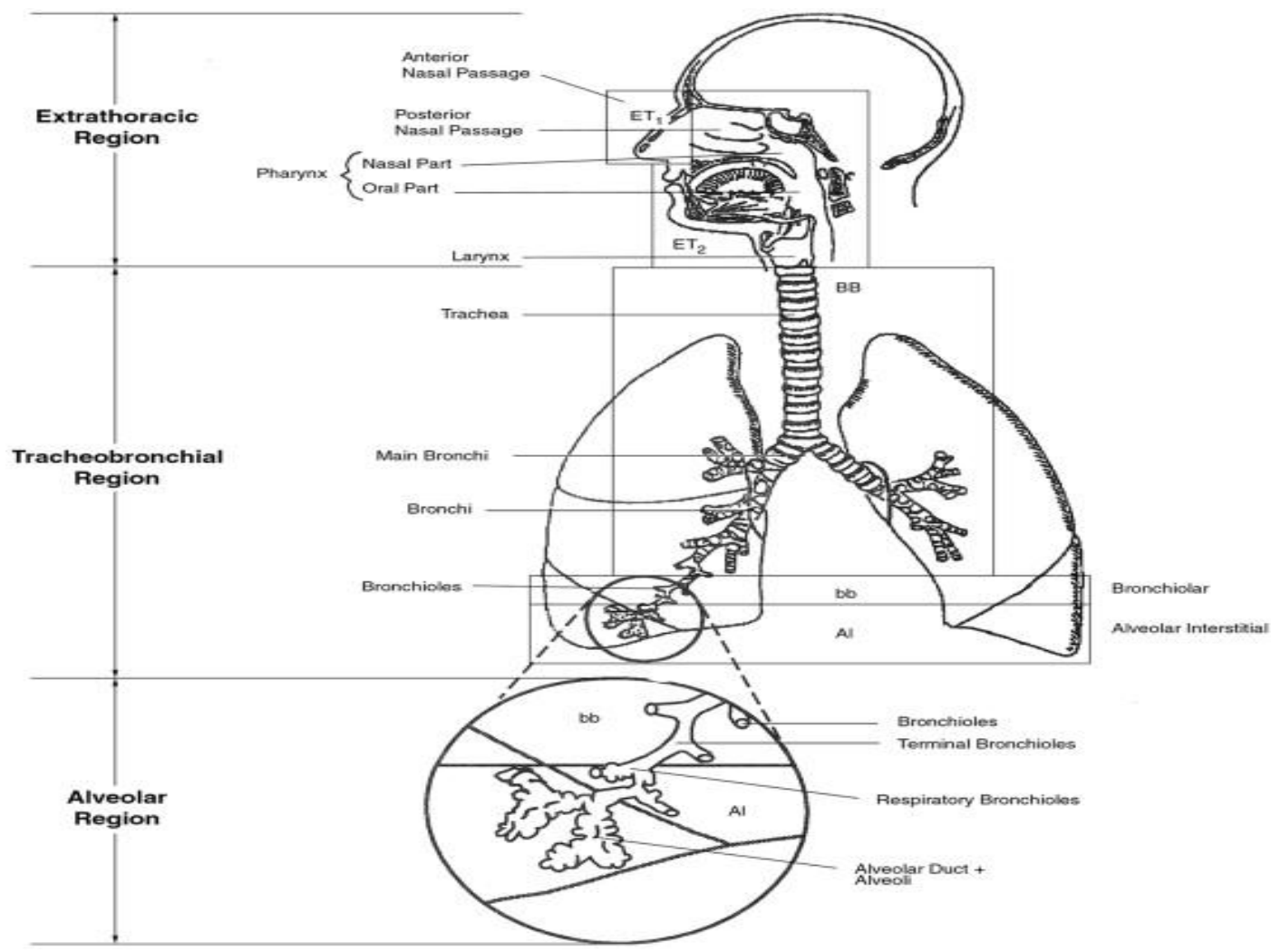

Figure (2): Regions of the respiratory tract model 
In the ET region, particle deposition is modeled by semi-empirical equations derived by Cheng [17]. Particle deposition fractions in bronchial and alveolar airways along each selected path are calculated by analytical equations for the simultaneous effects of diffusion, inertial impaction and sedimentation [15]. Resulting deposition rates are based on deposition fractions, atmospheric particle number concentration, breathing volume and breathing frequency. For the calculation of deposition density rates, mean surface areas of each airway generation were also calculated $[14,18]$.

In the present study, particle deposition was calculated for a Caucasian healthy adult male considering the following physical activity levels: sitting awake, light exercise and heavy exercise breathing [2]. Tidal volumes characteristic of sitting awake, light exercise and heavy exercise, corresponding breathing frequencies and resulting ventilation rates are listed in Table 1 . For heavy exercise breathing conditions, it was further assumed that the fraction of the total ventilatory airflow passing through the nose is only $50 \%$, while the rest is inhaled through the mouth [2].

\section{Results and Discussion}

Mass deposition fractions

Mass deposition fractions represent the fraction of inhaled mass which are deposited in a given region (or airway generation) of the respiratory tract during a single breath. Mass deposition fractions depend on mass size distribution Figure (1) and breathing parameters Table (1). Most of the particles are in the accumulation and coarse range, which mean that a large fraction will be filtered off in the ET region.

Regional (i.e. extrathoracic, bronchial, and acinar) and total mass deposition fractions and generational mass deposition fractions of $\mathrm{PM}, \mathrm{Pb}$ and $\mathrm{Fe}$ are plotted in Figs. (3a-3c), respectively for characteristic sitting, light exercise and heavy exercise breathing conditions. Table (2) summarizes mass deposition fractions (\%) of PM, $\mathrm{Pb}$ and $\mathrm{Fe}$ in the extrathoracic region (ET), bronchial region $(\mathrm{Br})$ and acinar region $(\mathrm{Ac})$ for an adult male under sitting, light and heavy physical activities

Most particles are deposited in the extrathoracic region for the three considered aerosols $(\mathrm{PM}, \mathrm{Pb}$ and $\mathrm{Fe}$ ), as shown in the left panels of Figure (3), due to the significant effect of impaction deposition mechanisms. The mass deposition fraction in ET region is significantly higher in case of light exercise than for heavy exercise breathing. This can be attributed to the switch off from $100 \%$ nasal breathing to $50 \%$ nasal/oral breathing with smaller deposition efficiencies for the oral route which means that more particles can reach the bronchial and acinar airways.

Table (2) indicates that the $\mathrm{Br}$ mass deposition fractions of PM represent 20\%, $11 \%$ and $29 \%$ of the ET deposition while the corresponding Ac mass deposition fractions of PM represent $56 \%$, $36 \%$ and $57 \%$ of the ET deposition for sitting, light and heavy physicsl activities, respectively. For $\mathrm{Pb}$, $\mathrm{Br}$, the mass deposition fractions represent $14 \%$, $7 \%$ and $19 \%$ of the ET deposition while those of Ac mass deposition fractions of PM represent $40 \%, 21 \%$ and $36 \%$ of the ET deposition for sitting, light and heavy physicsl activities, respectively. $\mathrm{Br}$ mass deposition fractions of $\mathrm{Fe}$ represent $23 \%, 13 \%$ and $28 \%$ of the ET deposition while Ac mass deposition fractions of Fe represent $55 \%, 38 \%$ and $64 \%$ of the ET deposition for sitting, light and heavy physicsl activities, respectively. Ac mass deposition is 2-3 times higher than $\mathrm{Br}$ deposition for the three aerosols.

Mass deposition fractions as a function of airway generation number increased with rising of the physical activity, and reached a maximum peak in the Ac airway generations in an interval of 19-22 for the three aerosols, as shown in right panels of Figure (3). This can be attributed to the significant effect of sedimentation deposition mechanism in lower airways. A shoulder peak (see the right panels), is observed in the $\mathrm{Br}$ region within an interval of 12-14 which is attributed to the significant effect of the particle impaction in the upper airways. These regions are the most important sites of lung from the point of view asthma [20].

\section{Mass deposition rates}

Mass deposition rates represent the mass deposited, in a given region (or airway generation) of the respiratory tract, per time unit. Mass deposition rates are based on the mass deposition fractions Figure (3). Total and regional (ET, Br, or Ac) mass deposition rates and generational mass deposition rates of PM, Fe and $\mathrm{Pb}$ are plotted in Figs. (4a- 4c), for characteristic sitting, light exercise and heavy exercise breathing conditions, respectively. 
Mass deposition rates increased with the increase of the physical activity in ET, Br and Ac regions of the lung [as shown in the left panels of Figs. (4ac)]. The significant increase of the mass deposition rates with increasing level of physical activity is due to the related increase of the ventilator rate from $0.54 \mathrm{~m}^{3} \mathrm{~h}^{-1}$ (for sitting conditions) to $3 \mathrm{~m}^{3} \mathrm{~h}^{-1}$ (for heavy exercise breathing conditions), as presented in Table (1). As with the deposition mass fraction, ET region has the highest deposition rates.

Mass deposition rates of $\mathrm{PM}, \mathrm{Pb}$ and $\mathrm{Fe}$ increased with the airway generations [as presented in the right panels of Figs. (4 a-c)] and a maximum peak is shown for the airway generations 19-22 in the Ac region of the lung, with the highest value for heavy exercise breathing conditions. A shoulder peak (see the right panels), is observed at generation number 13 of the $\mathrm{Br}$ region which is attributed to the significant effect of the particle impaction in the upper airways. It was found that the $\mathrm{Fe}$ mass deposition rates are higher than those of $\mathrm{Pb}$, which may be attributed to the high mass concentration of $\mathrm{Fe}$ compared to the one of $\mathrm{Pb}$. This indicates that the Ac region of the lung is a primary target for inhalation exposure.

Mass deposition rates of $\mathrm{PM}$ in the $\mathrm{Br}$ region represent $40 \%, 16 \%$ and $23 \%$ of the ET mass deposition rates while the corresponding mass deposition rates of $\mathrm{PM}$ in the Ac region represent $80 \%, 32 \%$ and $57 \%$ of the ET mass deposition rates under sitting, light and heavy physical activities, respectively.

Mass deposition rates of $\mathrm{Pb}$ in the $\mathrm{Br}$ region represent $33 \%, 17 \%$ and $20 \%$ of the ET mass deposition rates, while the corresponding mass deposition rates of $\mathrm{Pb}$ in the Ac region represent $67 \%, 21 \%$ and $40 \%$ of the ET mass deposition rates under sitting, light and heavy physical activities, respectively.

For Fe aerosol, Mass deposition rates of in the $\mathrm{Br}$ region represent $30 \%, 13 \%$ and $23 \%$ of the ET mass deposition rates, while the corresponding mass deposition rates of $\mathrm{Fe}$ in the Ac region represent $50 \%, 45 \%$ and $62 \%$ of the ET mass deposition rates under sitting, light and heavy physical activities, respectively. Mass deposition rates of the three aerosols in the Ac region is 2-3 times higher than the mass deposition rates in the Br region.

\section{Mass deposition density rates}

Mass deposition density rates represent the mass of inhaled particles deposited in a given region (or airway generation) of the respiratory tract per surface area unit and per time unit, which is equivalent to a "particle dose rate". This requires the calculation of mean surface areas for the different lung regions based on the stochastic lung model dimensions were obtained by integrating average generational surface areas within a given lung region $[14,18]$ Table (3).

Total and regional, i.e. extrathoracic (ET), bronchial $(\mathrm{Br})$ and acinar (Ac) mass deposition density rates and generational mass deposition rates of $\mathrm{PM}, \mathrm{Pb}$ and $\mathrm{Fe}$ are plotted in Figures $5 \mathrm{a}$ to $5 \mathrm{c}$, respectively for characteristic sitting, light exercise and heavy exercise breathing conditions.

Regional mass deposition density rates increased with the physical activity level from sitting to heavy exercise breathing conditions. ET mass deposition density rates has the highest value in comparison with the $\mathrm{Br}$ and $\mathrm{Ac}$ regions (see the left panels) as a result of exponentially increasing surface area from $0.045 \mathrm{~m}^{2}$ for the ET region to $145.58 \mathrm{~m}^{2}$ for the Ac region shown in Table (3).

Mass deposition density rates are distributed nonhomogenously (see the right panels) in the lung with a higher deposition in the bronchial airway generations. This indicates that the $\mathrm{Br}$ region of the lung is the primary target for health effects of inhaled particles. It is worth mentioning that lung cancers were observed in this region [19].

Table (1): Breathing parameters and ventilatory rates for the three applied breathing modes (ICRP, 1994)

\begin{tabular}{cccc}
\hline Breathing mode & $\begin{array}{c}\text { Tidal volume } \\
\left(\mathrm{cm}^{3}\right)\end{array}$ & $\begin{array}{c}\text { Breathing frequency } \\
\left(\mathrm{min}^{-1}\right)\end{array}$ & $\begin{array}{c}\text { Ventilatory rate } \\
\left(\mathrm{m}^{3} \mathrm{~h}^{-1}\right)\end{array}$ \\
\hline Sitting & 750 & 12 & 0.54 \\
Light exercise & 1250 & 20 & 1.5 \\
Heavy exercise & 1923 & 26 & 3 \\
\hline
\end{tabular}


Table (2): Mass deposition fractions (\%) of $\mathrm{PM}, \mathrm{Pb}$ and $\mathrm{Fe}$ in the extrathoracic region (ET), bronchial region (Br) and acinar region (Ac) for an adult male under sitting, light and heavy physical activities

\begin{tabular}{ccccccccccc}
\hline $\begin{array}{c}\text { Breathing } \\
\text { mode }\end{array}$ & \multicolumn{3}{c}{$\mathrm{PM}$} & \multicolumn{3}{c}{$\mathrm{Pb}$} & \multicolumn{3}{c}{$\mathrm{Fe}$} \\
\cline { 2 - 11 } & $\mathrm{ET}$ & $\mathrm{Br}$ & $\mathrm{Ac}$ & $\mathrm{ET}$ & $\mathrm{Br}$ & $\mathrm{Ac}$ & $\mathrm{ET}$ & $\mathrm{Br}$ & $\mathrm{Ac}$ \\
\hline Sitting & 25 & 5 & 14 & 35 & 5 & 14 & 22 & 5 & 12 \\
Light & 36 & 4 & 13 & 55 & 4 & 12 & 32 & 4 & 12 \\
Heavy & 28 & 8 & 16 & 42 & 8 & 15 & 25 & 7 & 16 \\
\hline
\end{tabular}
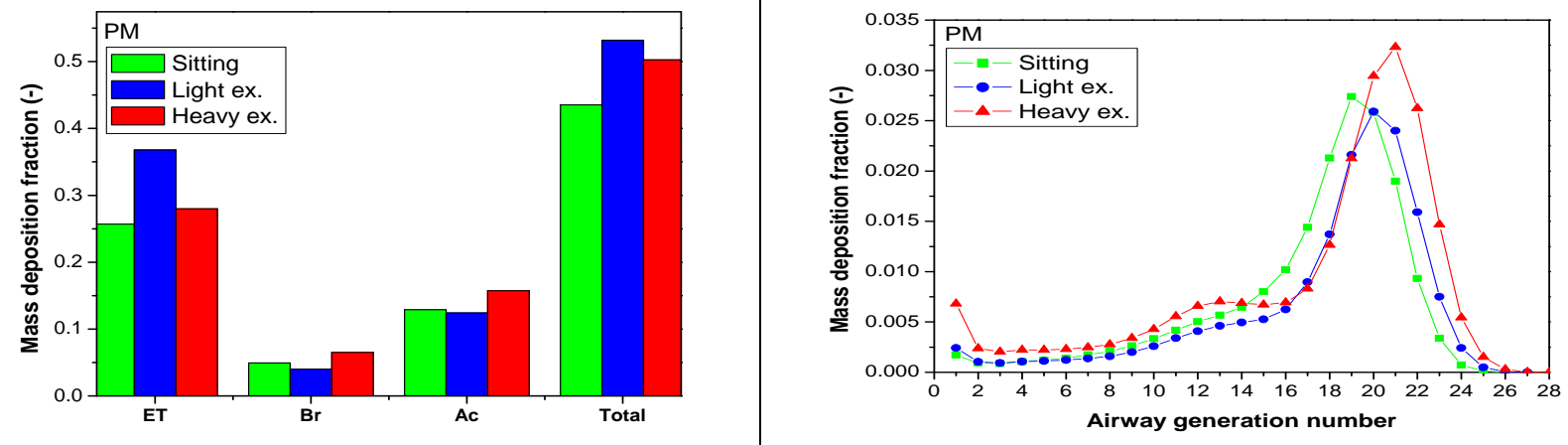

Figure (3a): Mass deposition fractions of particulate matter at regional (left panels) and airway generation (right panels) level for three characteristic breathing patterns in the respiratory tract

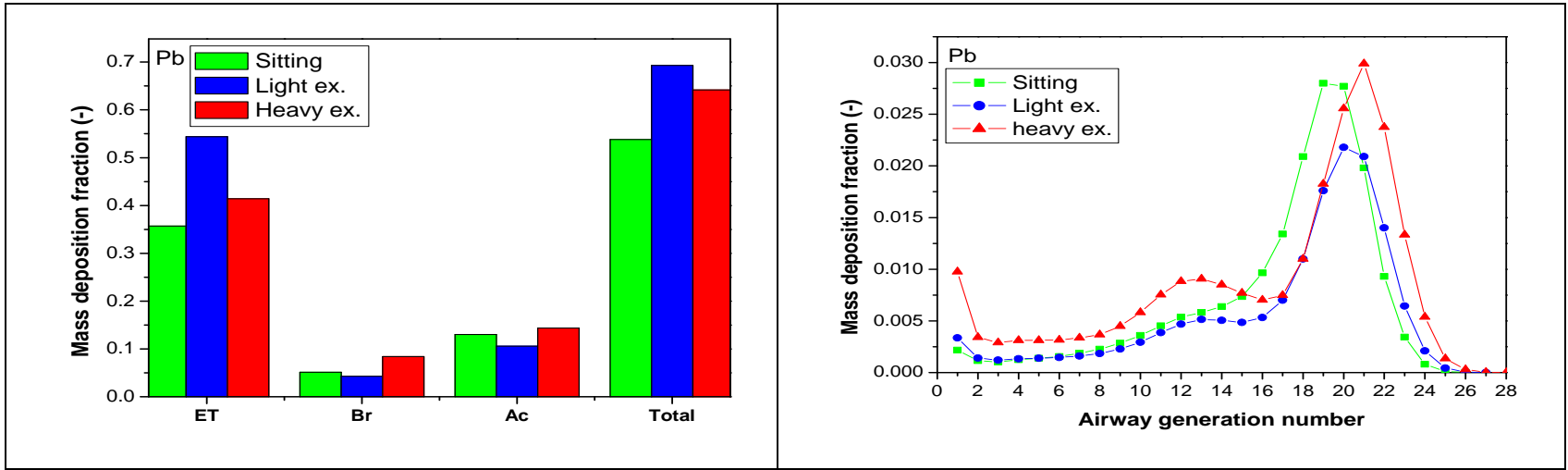

Figure (3b): Mass deposition fractions of $\mathrm{Pb}$ at regional (left panels) and airway generation (right panels) level for three characteristic breathing patterns in the respiratory tract 

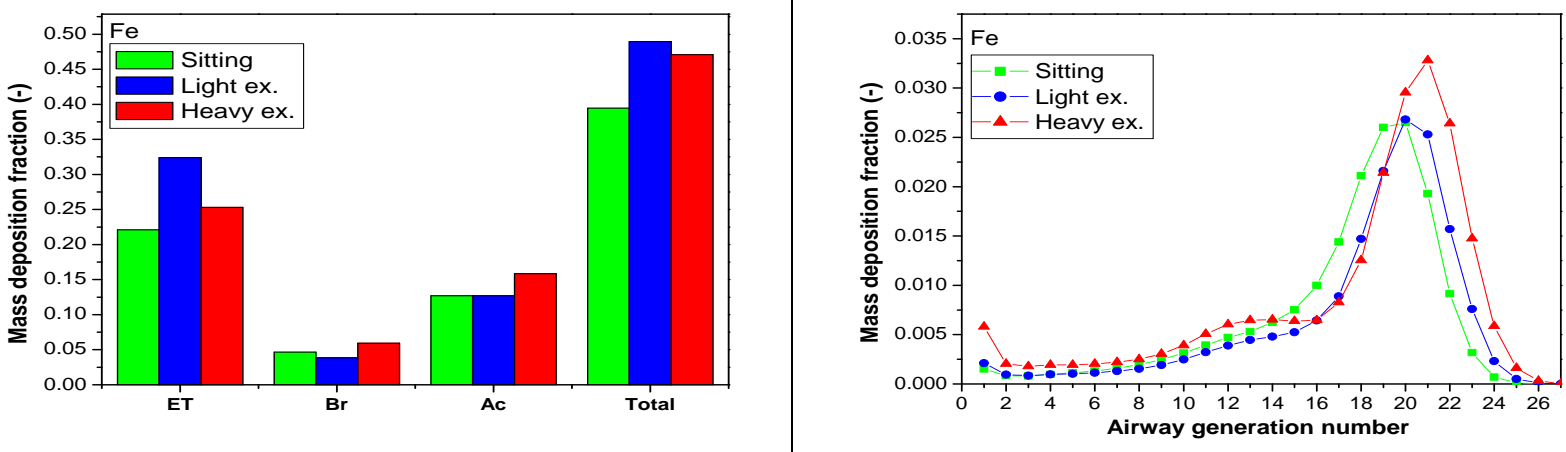

Figure (3c): Mass deposition fractions of Fe at regional (left panels) and airway generation (right panels) level for three characteristic breathing patterns in the respiratory tract
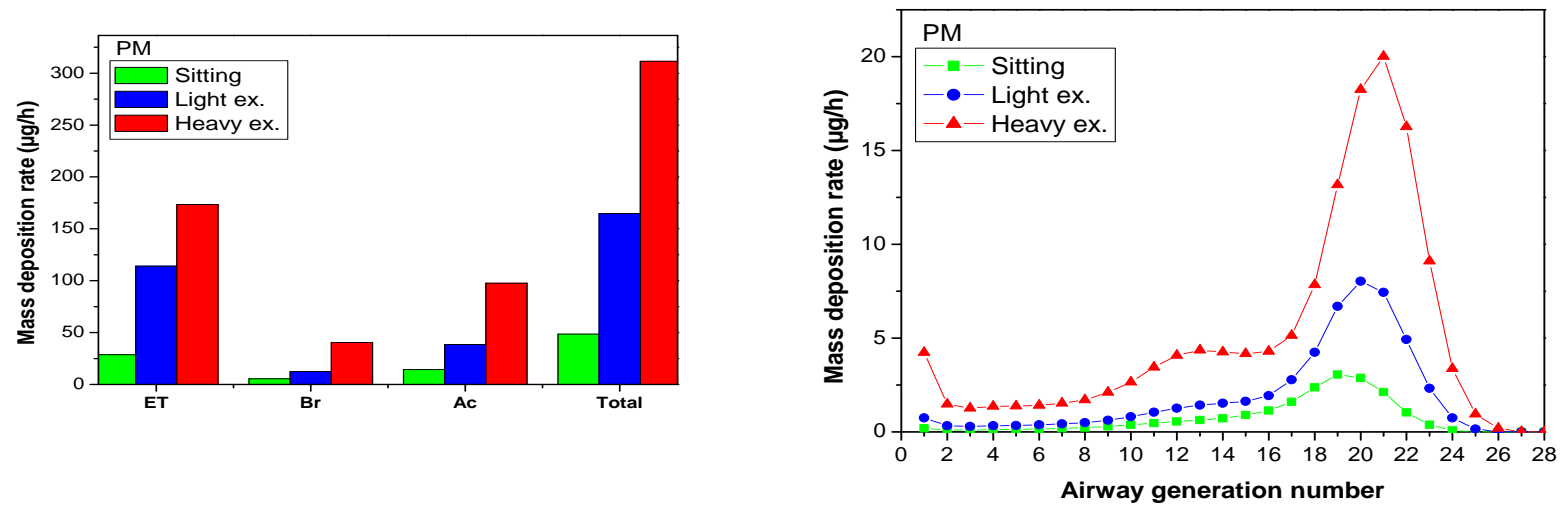

Figure (4a): Mass deposition rates of particulate matter at regional (left panels) and airway generation (right panels) level for three characteristic breathing patterns in the respiratory tract
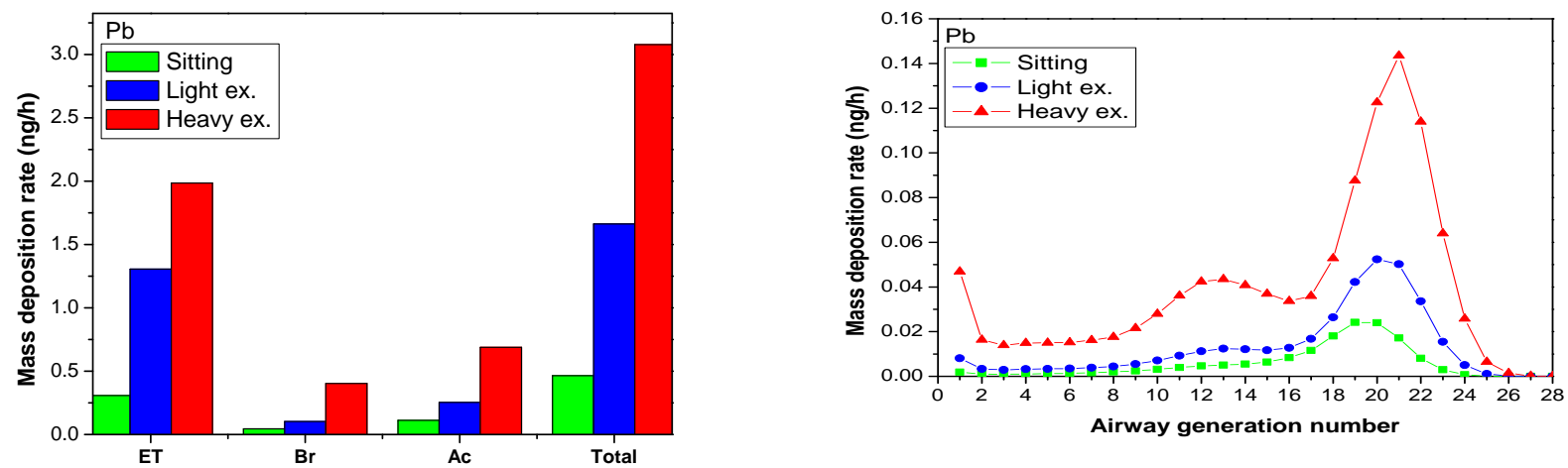

Figure (4b): Mass deposition rates of $\mathrm{Pb}$ at regional (left panels) and airway generation (right panels) level for three characteristic breathing patterns in the respiratory tract 

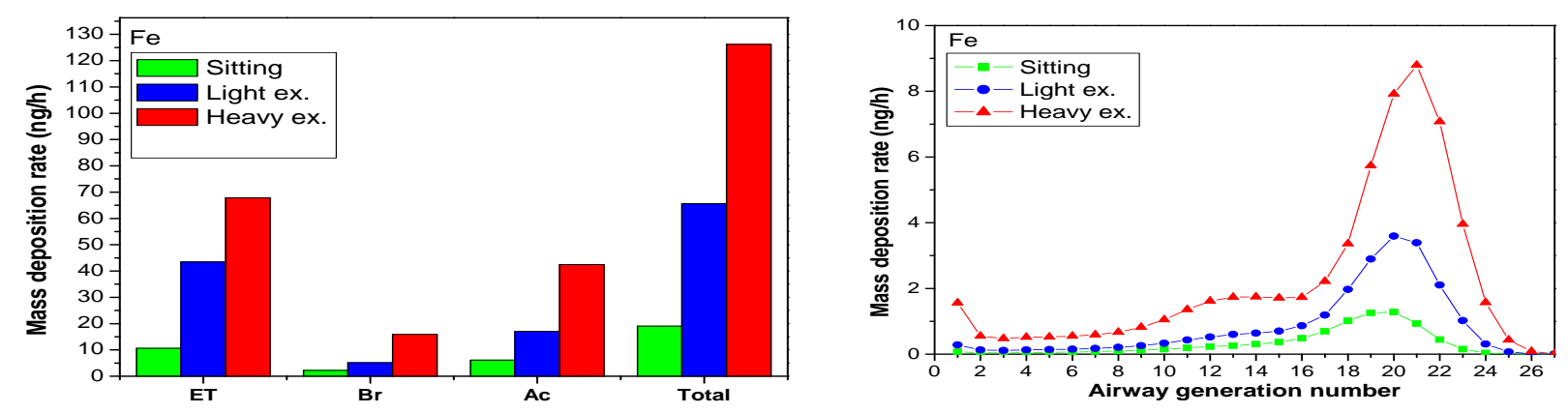

Figure (4c): Mass deposition rates of Fe at regional (left panels) and airway generation (right panels) level for three characteristic breathing patterns in the respiratory tract

Table (3): Total and regional surface area computed by the stochastic lung model

\begin{tabular}{cccc}
\hline \multicolumn{4}{c}{ Regional airway surface $\left(\mathrm{m}^{2}\right)$} \\
\hline Extrathoracic & Bronchial & Acinar & Total \\
0.0450 & 0.7534 & 145.5792 & 146.3776 \\
\hline
\end{tabular}
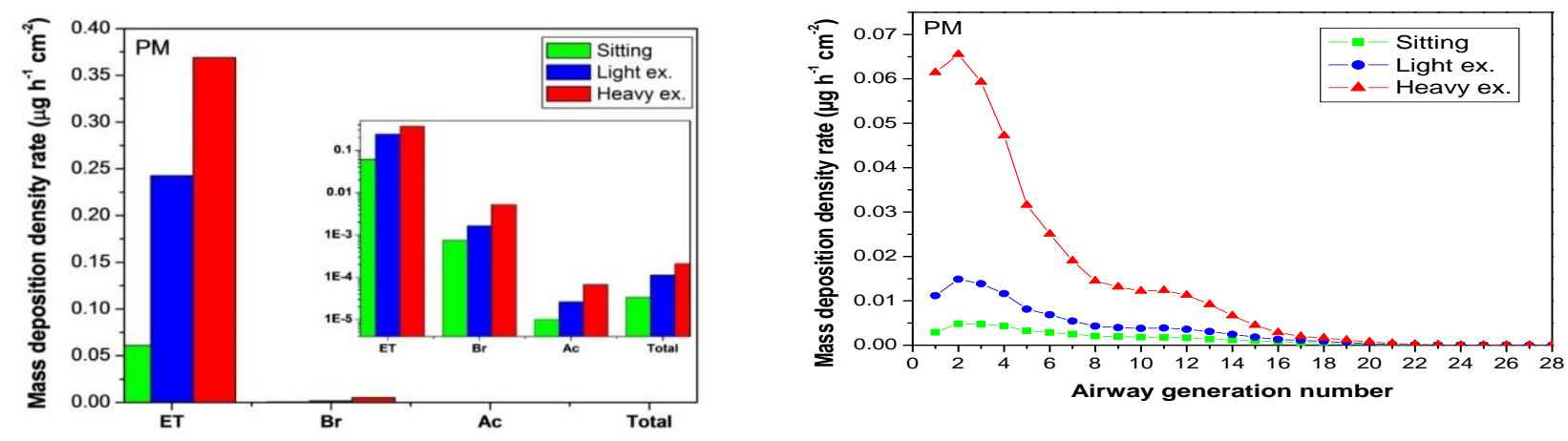

Figure (5a): Mass deposition density rates of particulate matter at regional (left panels) and airway generation (right panels) level for three characteristic breathing patterns in the respiratory tract 

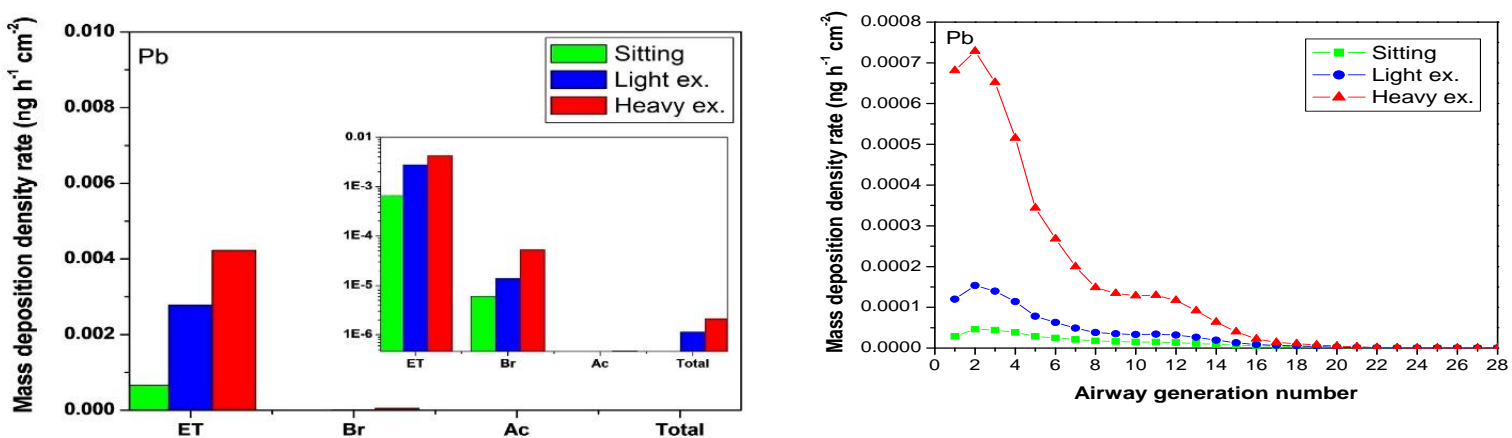

Figure (5b): Mass deposition density rates of $\mathrm{Pb}$ at regional (left panels) and airway generation (right panels) level for three characteristic breathing patterns in the respiratory tract
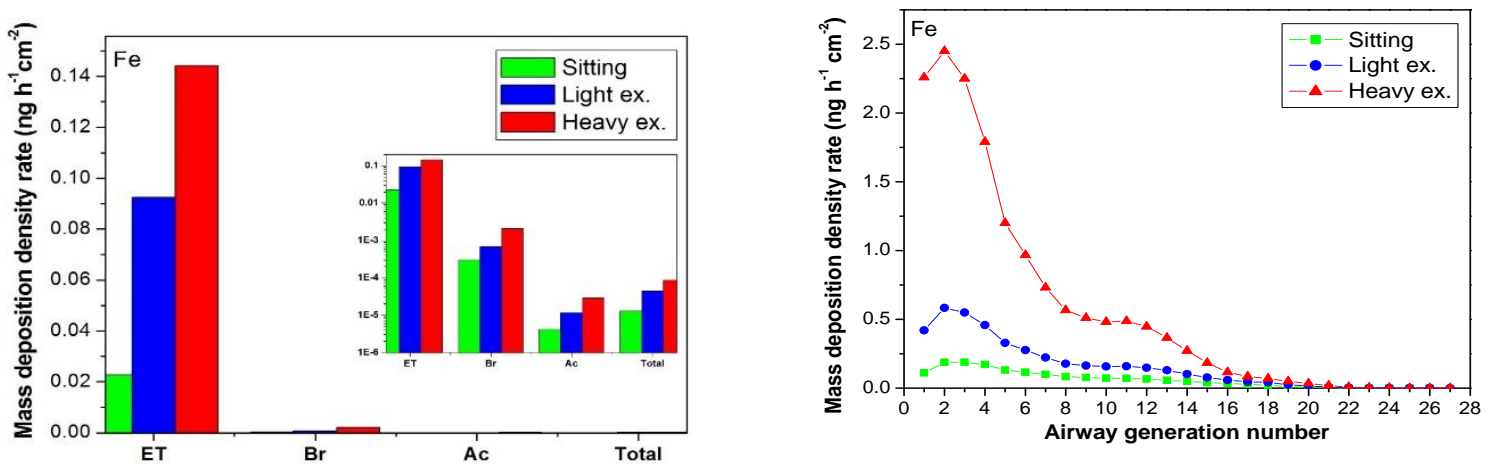

Figure (5c): Mass deposition density rated of Fe at regional (left panels) and airway generation (right panels) level for three characteristic breathing patterns in the respiratory tract

\section{Conclusions}

Potential dose metrics (mass deposition fraction, mass deposition rate and mass deposition density rate) for the estimation of health effects following the particles inhalation depends on the particle size and the breathing parameters of the respiratory tract. For the three considered aerosols (PM, Fe and $\mathrm{Pb}$ ), the highest deposition of the particles was observed in the ET region, while the lowest level of deposition was obtained in the $\mathrm{Br}$ region of the lung. The computations presented here clarify the significant role of physical activity on the particles deposition. The potential dose metrics increased with increasing level of physical activity from sitting to heavy exercise breathing conditions. Heavy physical activity leads to switching from nasal breathing to mixed nasal-and oral breathing which means more particles can reach the bronchial and acinar airways. A maximum peak was obtained in the airway generation of the Ac region in case of mass deposition fraction and mass deposition rates, while in the case of mass deposition density rates a maximum was observed in first airway generations of the $\mathrm{Br}$ region. The sites of bronchial and acinar deposition are the most important regions of the lung from the point of view of asthma. The present results have the same trend of deposition pattern and deposition sites in the lung airways of other studies. The results are to be further utilized for a risk assessment study.

\section{Acknowledgments}

The authors gratefully acknowledge the support given by the Faculty of Science, Minia University, Egypt for performing the experimental study. Acknowledgment is also conducted to prof. Werner Hofmann, Department of Chemistry and Physics of Materials, University of Salzburg, Austria, for his support throughout this work.

\section{References}

1- Spurny, K.R. (1994) Sampling, analysis, identification and monitoring of fibrous dusts and aerosols, Analyst, (119), 41-51. 
2- International Commission on Radiological Protection (ICRP). (1994) Human respiratory tract model for radiological protection, ICRP Publication 66. Ann. ICRP 24, Nos. 1-3.

3- Aryal, R., K., Aeri, Byeong-Kyu, L., Mohammad, K. and Simon, B. (2013) Characteristics of atmospheric particulate matter a nd metals in industrial sites in Korea, Environ. Poll., (2), 10-21.

4- Hu, X., Zhang, Y., Ding, Z., Wang, T., Lian, H., Sun,Y. and Wu, J. (2012) Bioaccessibility and health risk of arsenic and heavy metals $(\mathrm{Cd}, \mathrm{Co}, \mathrm{Cr}, \mathrm{Cu}, \mathrm{Ni}$, $\mathrm{Pb}, \mathrm{Zn}$ and $\mathrm{Mn}$ ) in TSP and PM2.5 in Nanjing, China, Atmos. Environ., (57), 146-152.

5- Pandey, P., Patel, D.K., Khan, A.H., Barman, S.C., Murthy, R.C. and Kisku, G.C. (2013) Temporal distribution of fine particulates (PM 2.5, PM 10), potentially toxic metals, PAHs and metal-bound carcinogenic risk in the population of Lucknow City, India, J. Environ. Sci. Health, Part A, (48), 730-745.

6- World Health Organization (WHO), Air Quality Guidelines for Europe. WHO Regional Publications. (2000) Regional Office for Europe, Copenhagen, Denmark. Eur. Ser.No91.

7- Samara, C. and Voutsa, D. (2005) Size distribution of airborne particulate matter and associated heavy metals in the roadside environment, Chemosphere, (59), 1197-1206.

8- Toscano, G., Moret, I., Gambaro, A., Barbante, C. and Capodaglio, G. (2011) Distribution and seasonal variability of trace elements in atmospheric particulate in the Venice Lagoon, Chemosphere , (85), 1518-1524.

9- Tran, D.T., Alleman, L.Y., Coddeville, Y.P. and Galloo, J.C. (2012) Elemental characterization and source identification of size resolved atmospheric particles in French classrooms, Atmos. Environ., (54), 250-259

10- Tsuda, A., Henry, F.S. and Butler, J.P. (2013) Particle transport and deposition: basic physics of particle kinetics, Comp. Physiol., (3), 1437-1471.

11- Oberdörster, G., Oberdörster, E. and Oberdörster, J. (2005) Nanotoxicology: an emerging discipline evolving from studies of ultrafine particles, Environ. Health Perspect., (113), 823- 839.
12- Nel, A., Xia, T., Maedler, L. and Li, N. (2006) Toxic potential of materials at the nanolevel, Science, (311), 622- 627.

13- Moustafa, M., Mohamed, A., Abdel-Rahman, A. and Hyam, N. (2015) Mass size distribution of elemental aerosol in industrial area, J. Adv. Res., (6), 827-832.

14- Balásházy, I., Alföldy, B., Molnár, A.J., Hofmann, W., Szöke, I. and Kis, E. (2007) Aerosol drug delivery optimization by computational methods for the characterization of total and regional deposition of therapeutic aerosols in the respiratory system, Curr. Computer-Aided Drug. Des., (3), 1332.

15- of particle-transport in a stochastic lung structure, J. Aerosol Sci., (21), 661-674.

16- Hofmann,W. and Koblinger, L. (1992) Monte Carlo modeling of aerosol deposition in human lungs: Part III. Comparison with experimental data, $J$. Aerosol Sci., (23), 51-63.

17- Cheng, Y.S. (2003) Aerosol deposition in the extrathoracic region, Aerosol Sci. Technol., (37), 659-71.

18- Salma, I., Balásházy, I., Hofmann,W. and Záray, G.Y. (2002) Effect of physical exertion on the deposition of urban aerosols in the human respiratory system, J. Aerosol Sci., (33), 983-997.

19- NRC (National Research Council) BEIR IV (1988) health risk of radon and other internally deposited alpha emitters, Washington, DC: National Academy Press.

20- Alföldy, B., Török, Sz., Balásházy, I., Hofmann,W. and Winkler-Heil, R. (2002) EPMA and XRF characterization of therapeutic cave aerosol particles and their deposition in the respiratort system, X-Ray Spectrum. (31),363-367.

21- Salma, I., Balásházy, I., Winkler-Heil, R., Hofmann,W. and Záray, G. (2002) Effect of particlemass size distribution on the deposition of aerosols in the human respiratory system, J. Aerosol Sci, (33) 119-132. 